\title{
Educação básica, capital humano e crescimento econômico: uma análise para os estados brasileiros ${ }^{1}$
}

\author{
Francisco Germano Carvalho Lúcio ${ }^{2}$ \\ José Weligton Félix Gomes ${ }^{3}$ \\ Ricardo Antonio de Castro Pereira 4
}

\begin{abstract}
Resumo: Este estudo analisa o impacto do capital humano no crescimento econômico dos estados brasileiros considerando a qualidade da educação básica na composição do capital humano. 0 trabalho analisa o período de 2005 a 2014, implicando a utilização de modelos de dados em painel. Para tanto, utilizou-se o modelo de Mankiw, Romer e Weil (1992) e o Índice de Desenvolvimento da Educação Básica - Ideb para inserir a qualidade da educação no capital humano. Visando abordar a heterogeneidade dos estados brasileiros e corrigir a heterocedasticidade e a autocorrelação detectadas, optou-se pela estimação do modelo com efeitos aleatórios utilizando mínimos quadrados generalizados (GLS). 0 modelo adotado mostrou-se adequado para o objetivo proposto.
\end{abstract}

Palavras-chave: Qualidade da educação. Capital humano. Crescimento econômico.

Classificação JEL: C23, H75, I25.

Basic education, human capital and economic growth: an analysis for the brazilian states

Abstract: This study intends to analyze the influence of human capital on the economic growth of the Brazilian states considering the quality of basic education in

1 Os autores agradecem as sugestões e as críticas dos pareceristas anônimos. Agradecem, ainda, o financiamento da Coordenação de Aperfeiçoamento de Pessoal de Nível Superior (CAPES) e do Conselho Nacional de Desenvolvimento Científico e Tecnológico (CNPq) e o apoio da Pró-Reitoria de Pesquisa e Pós-Graduação (PRPPG) da Universidade Federal do Ceará (UFC). Ricardo A. de Castro Pereira, adicionalmente, agradece o financiamento do Programa de Educação Tutorial (PET) da Secretaria de Educação Superior (Sesu) do Ministério da Educação (MEC) e o financiamento dos Institutos Nacionais de Ciência e Tecnologia (INCT) do CNPq.

2 Doutorando do Curso de Pós-Graduação em Economia (UFC/CAEN). E-mail: germanocarvalho15@hotmail.com.

3 Universidade Federal do Ceará - Campus de Sobral (UFC/Sobral). Pesquisador do Curso de PósGraduação em Economia CAEN-UFC. E-mail: weligtongomes@gmail.com.

4 Curso de Pós-Graduação em Economia CAEN, Universidade Federal do Ceará. E-mail: rpereira@caen.ufc.br. 
the composition of human capital. The paper addresses the period from 2005 to 2014, which implies to use panel data models. We used the model developed by Mankiw, Romer and Weil (1992) and the Basic Education Development Index (IDEB) to insert the quality of basic education in the human capital proxy. In order to capture the heterogeneity from the Brazilian states and to treat the detected heteroskedasticity and serial autocorrelation, we had chosen to use the random effects model with the Generalized Least Squares (GLS) method. The adopted model has shown adequate for the proposed objective.

Keywords: Education quality. Human capital. Economic growth.

\section{Introdução}

A partir da segunda metade do século passado o crescimento econômico passou a ser analisado através de modelos. Estes, associados a funções de produção que apontam os fatores determinantes para o crescimento. Solow (1956) criou um modelo de crescimento que utiliza uma função de produção do tipo Cobb-Douglas e preconiza uma convergência para um ponto no qual não há crescimento das variáveis em termos per capita, o estado estacionário.

Posteriormente o modelo de Solow apresentou algumas limitações analíticas, fato que gerou a concepção de outros modelos agregando melhoramentos que possibilitassem suprir suas deficiências. Assim, na década de 80 surgiram os modelos de crescimento endógeno, em especial com Romer (1986) e Lucas (1988). Tais modelos, dentre outros aspectos, consideram a importância do capital humano na modelagem e análises.

Muito embora o conceito de capital humano seja amplo no que tange à sua composição, atualmente os modelos de crescimento mais citados na literatura de teoria do crescimento econômico enfatizam educação como determinante do capital humano, como faz Barro (2013), por exemplo.

Ainda que outros fatores sejam necessários ao capital humano, considerar a educação como carro chefe serve de parâmetro norteador, tendo em vista as externalidades positivas geradas por esse fator e coniventes com seu resultado sobre o capital humano. Grossman (2006) afirma que a educação tem impacto sobre todas as atividades do indivíduo influenciando, por exemplo, a saúde. Essa é, pois, complementarmente, uma forma de capital humano. Há, porém, estudos que inserem separadamente proxies de capital humano e de saúde no modelo, como Firme e Simão Filho (2014).

A grande maioria dos estudos que combinam economia e educação utilizam variáveis de caráter quantitativo para representação da educação, sejam elas de fluxo ou de estoque, sendo 'anos de estudo' e 'taxas de matrícula' as mais comuns. No 
entanto, ultimamente tem-se buscado maior refino e acurácia de resultados utilizando variáveis que medem a qualidade do ensino. Corroborando o exposto acima Hanushek e Kimko (2000) analisam qualidade da educação e crescimento agregado verificando que a variável de qualidade da educação é estatisticamente significante. Os autores concluem que, ao analisar qualidade da educação, a variável que expressa quantidade perde poder explicativo.

Pereira (2004) verificou, pela análise da convergência do capital humano brasileiro entre os estados, que avanços têm sido obtidos em educação e em renda. Porém, dada uma diferença inicial nesse fator, ainda há a necessidade de reduzir a diferença de qualidade de vida entre as regiões e, como tal, entre os estados pertencentes às regiões mais desenvolvidas, a saber, Sul e Sudeste, vis-à-vis às menos desenvolvidas, Norte e Nordeste.

Este trabalho analisa o impacto do capital humano agregando qualidade na educação básica, no crescimento econômico dos estados brasileiros durante o período de 2005 a 2014. 0 direcionamento dado à qualidade da educação no presente estudo insere-o numa seara ainda com possibilidades amplas no Brasil devido à recente atenção em seu potencial analítico.

A composição das proxies, em especial a de capital humano, concede certa singularidade a este trabalho, além do fato de a análise desagregada para os estados brasileiros possuir poucas referências na literatura nacional. Foge do escopo deste análises referentes à convergência de renda entre os estados bem como comparações entre as proxies utilizadas e outras semelhantes.

0 presente estudo conta com mais cinco seções além desta introdução. A próxima seção expõe uma contextualização sobre a educação básica no Brasil. A seção 3 apresenta um referencial teórico dividido em subseções para melhor explanação da literatura. A seção 4 dispõe sobre a metodologia e os dados utilizados. A seção 5 apresenta os resultados obtidos por meio de análise de estatística descritiva e do modelo estimado, respectivamente. Por fim, a seção 6 apresenta algumas considerações como forma de conclusão do trabalho.

\section{Educação básica no Brasil}

O Brasil é um país territorialmente extenso. Se dividido em unidades menores, como regiões ou estados, suas discrepâncias de desempenho econômico podem ser melhor analisadas. Há vários motivos para tais diferenças, um deles apontado por Barros (2013) é a composição da população ao longo do tempo e os diferentes níveis educacionais que apresentaram a predominância desses grupos de povoamento em suas respectivas regiões. Apenas para fins de exemplificação aponta o perfil da população que povoou o Nordeste e o Sudeste. A região Sudeste tem um povoamento híbrido, com predominância de características da região Sul que recebeu imigrantes 
principalmente da Europa, que já chegaram ao Brasil com elevados níveis de escolaridade.

Já a região Nordeste teve seu povoamento alicerçado nas plantações de canade-açúcar com predominância de trabalho escravo de negros africanos e índios. Estes, contrariamente ao apontado para aqueles da região Sul e Sudeste, possuíam pouco ou nenhum nível de escolaridade. Diante disso a análise pode ser facilmente extrapolada para unidades menores, como no caso do presente estudo, os estados. Há que se frisar, porém, que essa diferença inicial garante apenas uma desvantagem exordial, a permanência ou aumento depende, em grande medida, da atenção voltada à educação. Assim, para Barros (2013) a persistência e/ou o aumento dessa situação de desigualdade educacional e de renda, até a atualidade, deve-se à inexistência de uma política de uniformização da educação.

Para Barbosa Filho e Pessôa (2009), a sociedade brasileira deu pouca, ou nenhuma, prioridade à acumulação de capital humano, em especial nos componentes educação e saúde, sobretudo no período do pós-guerra cujo pensamento era de que educação e saúde eram consequência e não causa do desenvolvimento econômico. Nesse segmento Barbosa Filho e Pessôa (2013) apontam que o modelo de substituição de importações adotado no Brasil priorizou o investimento em capital físico em detrimento a outros como as áreas sociais e, dentre elas, a educação.

Outro fator negativo para a baixa qualidade na educação brasileira foi a chamada transição demográfica, na qual, primeiramente, houve redução na taxa de mortalidade infantil numa sociedade com altas taxas de natalidade e depois uma redução na taxa de fecundidade. 0 resultado disso foi um crescimento populacional total acumulado de $410 \%$ num período de pouco mais de 70 anos. Aumentos esses, não acompanhados por investimentos sociais, dentre os quais, a educação.

Pessôa (2006) apresenta quatro pontos que julga de substancial importância ao entendimento da dinâmica da economia brasileira, dos quais, de forma conveniente, aqui serão considerados dois deles. Primeiramente, e abordando o conteúdo exposto até agora, menciona o atraso educacional como sendo um fator fundamental, provavelmente o maior, da baixa produtividade do trabalhador brasileiro. 0 segundo é a constatação do Brasil como um país onde a população pobre tem mais filhos do que a rica e com menor investimento em educação. Assim, aponta esses problemas como motivos que podem fazer essa diferença social permanecer por muito tempo.

Na década de 90 o Brasil demonstrou uma preocupação com a educação básica quando tentou universalizar o ensino fundamental. Pari passu a isso houve a transferência de sua gestão à esfera municipal. Segundo Leme, Paredes e Souza (2009) não foi possível identificar um efeito positivo em termos de melhoria de proficiência medida pelo Sistema de Avaliação da Educação Básica - Saeb. Porém, chama a atenção para a possibilidade da existência de efeitos positivos não captados.

Entretanto, e para finalizar esta seção, acerca da universalização supracitada Pessôa (2006) destaca que políticas que visem somente a abordagem quantitativa, 
nesse caso a universalização dos níveis da educação básica, não apresentam a eficiência necessária para alterar o cenário tal como se encontra. Devido a esse fato aponta uma necessidade de urgência no tocante ao problema da baixa qualidade educacional.

\section{Referencial teórico}

Dada a natureza de complementaridade entre os temas abordados no presente trabalho, a lembrar, educação, capital humano e crescimento econômico, uma análise dissociada torna-se deveras difícil. Todavia, objetivando uma melhor explanação desses temas, procurar-se-á, nas subseções a seguir, apresentar o referencial teórico de forma a apontar os tópicos contidos no tema pari passu apontamentos de relações entre si.

Inicialmente serão explanados alguns autores clássicos da temática capital humano bem como os modelos que predominaram na literatura do crescimento econômico na segunda metade do século passado. Em seguida, a análise consistirá em um apontamento da importância da relação entre qualidade na educação básica e capital humano. Deve-se frisar que a educação básica é ampla em termos de ciclos ou anos de estudo, que compreende desde os anos iniciais da vida escolar até o ensino médio.

\subsection{Capital humano e crescimento: os clássicos e os modelos}

Os estudos acerca do crescimento econômico receberam maior ênfase no século passado quando passaram a analisar o crescimento através de modelos. 0 modelo de Solow (1956) foi fundamental na literatura do crescimento econômico por associar uma função de produção do tipo Cobb-Douglas a algumas hipóteses simplificadoras e obter conclusões plausíveis acerca da aparente convergência de renda per capita entre os países.

As teorias e os modelos de crescimento a partir, cronológica e/ou teoricamente, do modelo supracitado apontam a importância da educação e do capital humano para o crescimento econômico. Mesmo que inicialmente o termo capital humano ainda não fosse amplamente difundido, percebe-se, claramente, como apontado em seguida, a preocupação com a importância na análise de tal fator.

Schultz (1961) argumenta que muito embora pareça óbvio que as pessoas adquiram habilidades e que estas sejam deveras úteis em suas vidas, não é tão óbvio que sejam uma forma de capital. Assim, salienta que muitos dos gastos considerados como consumo, por exemplo despesas com educação e saúde por exemplo, são na verdade investimento em capital humano. Há também a não contabilização do uso do tempo de lazer gasto para melhorar as habilidades e conhecimentos. Assim, essas e outras formas de captação do esforço humano podem melhorar a produtividade. Suas 
observações apontam para uma maior explicação pelo fator capital humano no aumento da produção nacional relativamente aos dos outros fatores de produção.

Nelson e Phelps (1966) chamam a atenção para os benefícios do trabalhador com maior nível de educação ao sugerirem que, em economias tecnologicamente progressistas ou dinâmicas, a gestão da produção é uma função que exige adaptação às mudanças. Assim, quanto mais educado um gerente é, mais rápida será a introdução de novas técnicas de produção. Em suma, a hipótese é de que pessoas com maiores níveis educacionais produzem inovações ou as implementam. Tem-se, pois, que a educação acelera o processo de difusão tecnológica.

Para inserir a discussão acerca do capital humano juntamente com os modelos de crescimento endógeno destaca-se Barro (1991) ao argumentar que o capital humano apresenta um papel importante nos modelos de crescimento endógeno e que esse tipo de capital é um insumo fundamental no setor de pesquisas, o qual gera ideias que contribuem para o progresso tecnológico. Este, por sua vez, impacta no crescimento econômico.

Romer (1986) em seu modelo de crescimento endógeno de longo prazo assume o conhecimento como um input da produção com aumentos de produtividade marginal. Assim, diferentemente dos modelos neoclássicos baseados em retornos marginais decrescentes, as taxas de crescimento podem aumentar no longo prazo.

Primeiramente, baseando-se na teoria do capital humano presente em Becker (1964) de que o crescimento é essencialmente guiado pela acumulação de capital humano, Lucas (1988) chamou atenção para a acumulação de capital humano como sendo uma alternativa para o crescimento sustentado. Além disso, distingue entre duas fontes possíveis de realizar essa acumulação, a saber, a educação e o learning by doing .

Sala-i-Martin (2000) mostra o modelo de Romer (1986) com externalidades do capital e direciona a análise seguindo a hipótese de Lucas (1988) de que a incidência das externalidades se dá sobre o estoque de capital per capita. 0 argumento para a existência dessas externalidades é a evidência do aumento da produção das empresas situadas próximas a outras que investem na produção. Romer (1986) aponta que uma empresa ao investir aumenta não só seu estoque de capital físico, mas também o seu nível de experiência e o seu conhecimento. Há, portanto, um processo conhecido na literatura afim como Knowledge Spill-overs ${ }^{6}$.

Mesmo com toda a importância advinda, sobretudo, do pioneiro modelo neoclássico de Solow e mesmo que este se mostre consistente com os dados estatísticos no tocante à evidência empírica da convergência de renda per capita entre

5 Aprender fazendo (Tradução própria). Termo utilizado na literatura para designar uma forma de aumento de produtividade aprendendo a utilizar melhor o equipamento disponível.

6 Transbordamento de conhecimento (Tradução própria). Termo utilizado na literatura para designar uma forma de implementação/uso de tecnologias sem, no entanto, investir o suficiente para gerar tal tecnologia. 
os países há uma diferença entre as estimações empíricas da participação do capital físico no produto com relação ao percentual necessário para a constatação da convergência supracitada. Alicerçado nessa diferença infere-se que o estoque de capital considerado no modelo neoclássico possui em sua composição, na verdade, pelo menos um outro fator, além do estoque de capital físico. Isso gerou a necessidade de uma adequação do modelo de tal forma que comportasse uma maior acurácia nesse tipo de análise.

Assim, o modelo desenvolvido por Mankiw, Romer e Weil (1992) surgiu da necessidade de ampliação do poder explicativo do modelo de Solow sem, no entanto, dispensar nem tampouco negligenciar suas funcionalidades e seu potencial explicativo, sobretudo no que tange às questões de análise de convergência. 0 modelo de Mankiw, Romer e Weil (1992), à grosso modo, insere um componente que representa capital humano ${ }^{7}$, de forma explícita, no modelo de Solow tradicional. Um conveniente detalhamento desse modelo dar-se-á apenas na seção Metodologia.

Muito embora toda a teoria mostrada até agora convirja para um consenso de que capital humano influencia positivamente o crescimento econômico, alguns estudos apresentaram resultados contrários à teoria. Nakabashi e Figueiredo (2008) e Marquetti, Berni e Hickmann (2002) tanto apontam alguns trabalhos cujos resultados divergem da teoria ${ }^{8}$ quanto apontam Temple (1999) como exemplo de trabalho que justifica a não conivência daqueles com a teoria.

Temple (1999) mostra as possíveis falhas de especificação e/ou o trato dos dados por parte daqueles que obtém resultados contrários à teoria. Segundo Temple (1999, 2001) alguns outliers da amostra podem mudar o resultado de forma significativa. Assim, dada a característica desses pontos muito destoantes da média, pode ser perigoso generalizar amplas regressões de crescimento sem, no entanto, investigar de forma cautelosa o grau de heterogeneidade dos parâmetros.

\subsection{Qualidade na educação e capital humano}

O foco na qualidade da educação como composição do capital humano é relativamente recente. Segundo Nakabashi e Figueiredo (2008) são minoria os que enveredam por esse enfoque. Como explicitado anteriormente, este trabalho tenciona utilizar a qualidade da educação como composição de uma proxy para capital humano. Assim, vê-se conveniente destacar alguns estudos que corroborem o objetivo proposto.

Hanushek e Kimko (2000) apontam uma forte relação entre a qualidade da educação e o crescimento da renda per capita. Barro (2013) utilizando testes

7 A chamada Proxy de Mankiw, Romer e Weil (1992) consiste no produto a fração da população matriculada no ensino secundário e a população em idade de estar no ensino secundário dividido pela população economicamente ativa.

8 Ver, por exemplo, Benhabib e Spiegel (1994) e Pritchett (1996). 
internacionais de proficiência, como proxies, para qualidade do capital humano encontra uma relação positiva entre os testes e as taxas de crescimento da renda real per capita.

Connolly (2004) utilizando dados em painel para 48 estados americanos separados em cinco regiões, tendo como amostra temporal os anos de 1880, 1900, 1920 e 1950 analisa os gastos reais anuais em educação como proxy para qualidade do capital humano e encontra resultados semelhantes àqueles apontados no parágrafo anterior. Embora tenha obtido êxito nos resultados, tal proxy não necessariamente obterá os mesmos resultados em outros países, uma vez que se deve considerar a eficiência nesses gastos. Essa eficiência varia quando da mudança da unidade geográfica analisada fazendo variar também a qualidade da educação nessa respectiva unidade.

Visando inserir qualidade na educação na análise de capital humano Nakabashi e Figueiredo (2005) utilizam a porcentagem da população em idade de trabalhar que está matriculada no ensino secundário como proxy para capital humano. Porém, há diferenças na qualidade da educação nas diversas unidades da federação. Logo, os indivíduos devem apresentar diferentes níveis de capital humano em diferentes unidades geográficas mesmo estando matriculados no mesmo nível.

Seguindo a linha de inserir qualidade do ensino na composição do capital humano Nakabashi e Figueiredo (2008) propõem um método de mensuração das proxies para capital humano que contemple aspectos qualitativos e quantitativos. Tal método consiste no produto entre a proxy de Mankiw, Romer e Weil (1992), o Índice de Desenvolvimento Humano - IDH e o IDH ao quadrado. "A suposição por trás da utilização dessa proxy é que a qualidade do sistema educacional depende do nível de desenvolvimento da unidade geográfica em questão, sendo o IDH a variável utilizada para tal mensuração". (NAKABASHI; FIGUEIREDO, 2008, p. 9).

Muito embora os resultados obtidos por Nakabashi e Figueiredo (2008) tenham sido satisfatórios ao objetivo do estudo de apontar sua proxy como tendo maior poder de ajustamento vis-à-vis aquela utilizada por Mankiw, Romer e Weil (1992), apontam uma série de problemas que a inserção do IDH no cálculo pode acarretar como endogeneidade, por exemplo. Essa endogeneidade é, sobretudo, devida ao fato de se querer explicar a renda ao passo que a renda é um dos componentes do IDH. Descartase, pois, no presente estudo a possibilidade de utilização das proxies apontadas acima.

Soares (2009) utiliza o Índice de Desenvolvimento na Educação Básica - Ideb para inserir qualidade da educação básica numa proxy para capital humano. Tal proxy é obtida pela multiplicação do número de anos médios de estudo da população com mais de 25 anos pelo Ideb tendo como recorte os municípios brasileiros. Aqui, seguiuse Soares (2009) e utilizou-se o Ideb como componente da proxy que representa a qualidade da educação. Porém, o Ideb referente apenas ao ensino médio. Já para o componente que representa a quantidade utilizou-se o dado referente aos anos de estudo da População Economicamente Ativa - PEA. 


\section{Metodologia}

O intuito do presente estudo de incluir o capital humano como fator explicativo do crescimento gera a necessidade de utilização de um modelo que explicite a utilização desse fator em sua composição. Para tanto, utilizar-se-á o modelo de Mankiw, Romer e Weil (1992). Há que se frisar que Mankiw, Romer e Weil (1992) não discordam dos parâmetros utilizados do modelo de Solow. Nesse sentido, Firme e Simão Filho (2014. p. 5) ao se referirem ao modelo de Solow observam: "parece que a magnitude dos termos foi subestimada no modelo original". Em outras palavras, pretende-se obter um maior refino na análise dos resultados com a desagregação do estoque de capital em capital físico e humano.

Devido ao teor de desagregação espacial da análise aqui proposta, por estados brasileiros, juntamente ao período considerado, a lembrar, de 2005 a 2014, há a necessidade de a estimação ser realizada através da utilização de um modelo de dados em painel. Por estudar repetidamente um corte transversal, tal escolha transcende o caráter da análise estática e apresenta a vantagem de melhor adequar-se ao estudo da dinâmica da mudança, como destacado em Gujarati (2006).

A combinação de cortes transversais com séries temporais gera um número maior de observações, que por sua vez, apresentam mais graus de liberdade. Modelos de dados em painel apresentam, portanto, maior eficiência comparativamente às análises realizadas por meio de modelos de séries temporais ou cortes transversais puros. A forma na qual o modelo será estimado depende das relações desempenhadas entre as variáveis envolvidas e, além disso, das relações destas com os termos de erro.

Em suma, dados em painel apresentam inúmeras vantagens, dentre as quais Kennedy (2008) destaca: (i) a possibilidade de tratamento da heterogeneidade não observada entre as unidades cross-section, evitando assim problemas de endogeneidade e, consequentemente, viés dos estimadores; (ii) a presença de uma maior variabilidade nos dados através da combinação de dados cross-section com variação ao longo do tempo; (iii) a possibilidade de estudar fenômenos que os dados cross-section e de séries de tempo não realizam de maneira isolada; e (iv) uma melhor análise de ajustamento dinâmico dos fenômenos econômicos de longo prazo.

Modelos que utilizam dados em painel podem ser estimados de formas distintas. As possibilidades aqui consideradas são estimações por Mínimos Quadrados Ordinários, no caso de modelo restrito (Pooled), por Mínimos Quadrados Generalizados, e os modelos de efeitos fixos e de efeitos aleatórios. A escolha não depende do perfil do pesquisador, nem tampouco se mostra explicitamente quando da escolha das variáveis. Tal escolha pode ser auxiliada através da aplicação de alguns testes adequados a essa finalidade.

Este estudo seguirá a maioria das pesquisas que utilizam a metodologia de dados em painel e utilizará um painel equilibrado, isto é, em que todas as unidades de 
observação de cortes transversais contenham o mesmo número de observações de séries temporais. A série temporal do painel utilizado consta de 10 anos, ou seja, dados anuais de 2005 a 2014. Para tanto, algumas séries de dados devem ser ajustadas devido a não disponibilidade de alguns dados. As duas subseções que seguem tratam do modelo e dos dados, respectivamente.

\subsection{Modelo}

Como já destacado anteriormente, Mankiw, Romer e Weil (1992) estenderam o modelo de Solow tradicional ${ }^{9}$ e incluíram de forma explícita um componente que representa o capital humano, aqui denotado pela letra $(H)$. Conforme Firme e Simão Filho (2014), a inclusão dessa variável reduz outros efeitos como o da poupança e do crescimento populacional sobre a renda. 0 modelo expandido pelos autores foi capaz de explicar 80 \% da variação da renda per capita entre os países. 0 modelo de Mankiw, Romer e Weil (1992) apresenta-se em função de quatro fatores. Como segue:

$$
Y_{t}=F\left(A_{t}, K_{t}, H_{t}, L_{t}\right)
$$

A fim de facilitar o entendimento do leitor seguiu-se a notação padrão da literatura, onde $Y_{t}$ é o Produto; $A_{t}$ representa o parâmetro tecnológico, ou de produtividade; $K_{t}$ denota o capital físico; $H_{t}$ é o capital humano; e $L_{t}$ o fator trabalho. A equação (1) mostra tão somente a notação da variável dependente em função dos fatores de produção. Por outro lado, a equação (2) mostra a forma funcional da função de produção do tipo Cobb-Douglas:

$$
Y_{t}=K_{t}^{\alpha} H_{t}^{\beta}\left(A_{t} L_{t}\right)^{(1-\alpha-\beta)}
$$

Além das variáveis descritas anteriormente, na equação (2) temos $\alpha$ e $\beta$ com valores entre 0 e 1 , que representam as elasticidades do capital privado e do capital humano em relação ao produto. Logo, os expoentes $\alpha, \beta$ e $(1-\alpha-\beta)$ representam as parcelas de cada um dos fatores de produção no produto. 0 parâmetro tecnológico é disposto juntamente com o fator trabalho devido à característica da tecnologia de proporcionar maior produtividade a ele.

A metodologia utilizada implica que a equação a ser estimada deve atender a característica de linearidade nos parâmetros. Logo, o modelo especificado em (2) pode ser linearizado através da aplicação do logaritmo natural. 0 processo de linearização concede ao modelo a seguinte forma:

9 O modelo de Solow dito tradicional, fundamentado em Solow (1956), utiliza como fatores de produção em uma função de produção do tipo Cobb-Douglas apenas capital físico e trabalho. 


$$
\operatorname{Ln} Y_{t}=\alpha \operatorname{LnK} K_{t}+\beta \operatorname{Ln} H_{t}+(1-\alpha-\beta) L n L_{t}+(1-\alpha-\beta) \operatorname{Ln} A_{t}
$$

Seguindo a hipótese de que o parâmetro de produtividade é constante, temos que o último termo da equação (3) será o intercepto, isto é, o termo a constante da função estimada. Além disso, compatibilizando a equação (3) para a metodologia de dados em painel, temos:

$$
\begin{gathered}
\operatorname{Ln} Y_{i t}=\beta_{0 i t}+\beta_{1 i} L n K_{i t}+\beta_{2 i} L n H_{i t}+\beta_{3 i} \operatorname{Ln} L_{i t}+\varepsilon_{i t} \\
\text { Onde } \beta_{0 i t}=(1-\alpha-\beta) \operatorname{Ln} A, \beta_{1 i}=\alpha, \beta_{2 i}=\beta \text { e } \beta_{3 i}=1-\alpha-\beta \mathrm{e} \\
t=2005,2006, \ldots, 2014 .
\end{gathered}
$$

Segundo Hsiao (1986), os modelos de dados em painel, tal como especificado pela equação (4), oferecem inúmeras vantagens em relação aos modelos de corte transversais e de séries temporais, pois controlam a heterogeneidade presente nas unidades estudadas, nesse caso os estados brasileiros. Ademais, a omissão das variáveis que representam a heterogeneidade pode provocar um viés dos estimadores.

Ressalta-se, ainda, que o uso de dados em painel permite controlar os efeitos das variáveis não observadas e, portanto, as diferenças existentes entre os estados brasileiros. 0 procedimento para a estimação de modelos de dados em painel depende, portanto, do tratamento dado ao parâmetro $\beta_{0 i t}$. Esse parâmetro capta as diferenças existentes entre as unidades do painel.

De acordo com Hill, Griffiths e Judge (1999) o modelo de efeitos fixos é utilizado quando existe a necessidade de se controlar os efeitos de variáveis omitidas no modelo e que são invariantes ao longo do tempo. Nesse caso o intercepto $\beta_{0 i t}$ será igual a $\beta_{0 i}$ e $\beta_{1 i t}=\beta_{1}, \ldots, \beta_{k i t}=\beta_{k}$. Ou seja, difere entre as unidades do painel, porém não variam com o tempo. Sendo assim, o modelo apresentado na equação (4) dá-se por:

$$
\operatorname{Ln} Y_{i t}=\beta_{0 i}+\beta_{1} \operatorname{Ln} K_{i t}+\beta_{2} L n H_{i t}+\beta_{3} L n L_{i t}+\varepsilon_{i t}
$$

Onde $\beta_{0 i}$ é o intercepto que capta as diferenças entre os indivíduos.

No modelo de efeitos aleatórios o intercepto do modelo é tratado como uma variável aleatória extraída de uma população maior de indivíduos. Nesse modelo as variáveis explicativas não podem estar correlacionadas com o termo de erro aleatório. Hill, Griffiths e Judge (1999) modelam os $n$ interceptos do modelo de dados em painel com efeitos aleatórios considerando $\beta_{0 i}=\beta_{0}+\alpha_{i}$. Desta forma tratam o 
intercepto em duas partes, sendo que uma parte representa o comportamento dos indivíduos $\left(\alpha_{i}\right)$, tal como no modelo de efeitos fixos, porém agora sendo tratado como aleatório, e a outra parte invariante $\left(\beta_{0}\right)$, correspondente ao intercepto populacional.

Portanto, partindo novamente da equação (4), o modelo de dados em painel com efeitos aleatórios seria dado da seguinte forma:

$$
\begin{gathered}
L n Y_{i t}=\beta_{0}+\beta_{1} L n K_{i t}+\beta_{2} L n H_{i t}+\beta_{3} L n L_{i t}+v_{i t} \\
\text { Em que } v_{i t=} \alpha_{i}+\varepsilon_{i t} .
\end{gathered}
$$

\subsection{Especificação das variáveis e dados}

0 modelo especificado na subseção anterior requer a utilização das variáveis, ou das suas respectivas proxies, para cada estado brasileiro. 0 modelo utiliza o produto $(Y)$, o estoque de capital físico $(K)$, a força de trabalho $(L)$, o estoque de capital humano $(H)$ e o parâmetro tecnológico $(A)$. A escolha do período, a lembrar, de 2005 a 2014, justifica-se pela disponibilidade de informações; ainda que extrapolações tenham sido necessárias para equilibrar o painel, estas foram feitas de forma moderada para não comprometer o potencial analítico. Para fins de melhor entendimento segue a descrição de cada uma das variáveis utilizadas com suas respectivas denominações neste estudo.

i) PIB: Corresponde ao Produto Interno Bruto - PIB estadual. Os dados serão coletados dos relatórios Contas Regionais do Brasil, do Instituto Brasileiro de Geografia e Estatística - IBGE (2011, 2014b, 2016). Os valores foram deflacionados utilizando o Índice de Preços ao Consumidor Amplo - IPCA, com base no ano de 2005. Os valores referentes a essa variável estão na escala de milhões.

ii) CAP_FIS: Corresponde ao estoque de capital físico de cada unidade da federação. Dada a ausência de uma medida precisa que aponte o estoque de capital, surge a necessidade da utilização de uma proxy. Segundo Firme e Freguglia (2013) "é muito difícil obter uma boa proxy para capital físico". Assim, como é frequente na literatura, por exemplo em Tavares, Ataliba e Castelar (2001), utilizar-se-á neste estudo o consumo de energia elétrica não-residencial. Mesmo que hajam críticas acerca dessa proxy, como em Barretto e Mitrulis (2001) ao afirmarem que avanços tecnológicos podem reduzir o uso de energia elétrica mesmo que o estoque de capital físico permaneça constante, optou-se pela citada supondo um melhor ajustamento em relação a outras frequentemente utilizadas como 'Consumo de Energia do Setor Industrial' devido ao fato de considerar outros setores da economia. Esta é medida em Kilowatts/hora (Kw/h).

Com base em dados sobre o 'consumo total' e o 'consumo residencial', desagregado por estados, a proxy utilizada (Consumo de Energia Não-residencial) foi 
obtida por complementaridade. 0 consumo total de energia foi obtido do Anuário Estatístico de Energia Elétrica (2011, 2013, 2015). Já o consumo residencial foi obtido no Ipeadata ${ }^{10}$. Para o ano de 2005, o 'Consumo Total' foi obtido via extrapolação ${ }^{11}$ fundamentada na média de crescimento do restante do período considerado no estudo, ou seja, de 2006 a 2014.

iii) Trabalho: Representa a força de trabalho. Aqui, utilizar-se-á como proxy a População Ocupada. Esta é composta pelo produto da porcentagem de indivíduos ocupados, com base nos dados coletados na Pesquisa Nacional por Amostra de Domicílios - PNAD e IBGE (2014a), multiplicada pela população residente ${ }^{12}$ de cada estado, a partir do IBGE. Tal cálculo faz-se necessário devido ao caráter amostral da PNAD. 0 ano de 2010 foi obtido via interpolação, tendo em vista que não houve PNAD no referido ano. Optou-se pela interpolação ao invés de utilizar os dados do Censo 2010, visando não utilizar dados de bases diferentes e, consequentemente, metodologias diferentes na mesma série de tempo.

iv) CAP_HUM: Representa o estoque de capital humano. Dada a discussão apresentada no referencial teórico acerca da inserção da qualidade da educação na proxy de capital humano, neste estudo será elaborada uma proxy que agregue ambos, qualidade e quantidade de educação. A composição dar-se-á pela multiplicação entre a variável 'Anos de Estudo' da população economicamente ativa - PEA ${ }^{13}$, rotulada pela literatura por ser uma medida apenas de quantidade, e uma proxy que capte a qualidade do ensino. Aqui utilizar-se-á o Índice de Desenvolvimento da Educação Básica - Ideb ${ }^{14}$, referente a terceira série do Ensino Médio sob o argumento de que este é o nível de capital humano no qual o indivíduo deixa o educação básica e está apto, no sentido legal, para o mercado de trabalho. Soares (2009) utiliza o Ideb como ponderação, porém, seu trabalho é um crosssection para o ano de 2005, além de a análise direcionar-se a unidades geográficas menores, a saber, municípios. Ainda que o Ideb seja lançado bianualmente, a proxy de capital humano concebida neste estudo será diferente em cada ano da série de 2005 a 2014 devido ao fato de que seu componente quantitativo se altera a cada ano. Frisa-se que o principal motivo para a elaboração da variável de capital humano, tal como descrito acima, foi a tentativa de reduzir o viés de endogeneidade causado pela variável anos de estudo isoladamente.

10 Disponível em: http://www.ipeadata.gov.br/Default.aspx

110 procedimento utilizado foi a subtração da taxa média de crescimento dos valores referentes aos anos disponíveis, de 2006 a 2014, do valor referente ao ano de 2006. Para a obtenção da taxa média de crescimento primeiramente calculamos a taxa de crescimento anual e, após isso, a média destas.

12 Ao utilizar a 'População residente' no cômputo, capta-se melhor o resultado líquido de fluxos migratórios.

13 Os dados dessa variável foram coletados no site do IBGE. Disponível em: https://www.ibge.gov.br/

14 Disponível em: http://ideb.inep.gov.br/resultado/resultado/resultado.seam?cid=1211699 
v) Const:: Representa o nível tecnológico, ou de produtividade, presente na função de produção mostrada nas equações (2) e (3). Muito embora Tavares, Ataliba e Castelar (2001) considerem a denominação de nível tecnológico como inapropriada manter-se-á, aqui, para fins de simplificação. Este é, por hipótese, constante.

Como de praxe, aponta-se como mais indicado trabalhar o modelo com dados da mesma base devido à homogeneidade destes quando coletados. Contudo, como na maioria dos casos isso não é possível devido à natureza das variáveis. 0 modelo aqui utilizado precisa de variáveis com características diferentes e não há, para o recorte proposto, uma base que contenha todas as variáveis escolhidas. Além disso, dada a natureza do estudo e das proxies utilizadas, bem como o período analisado, há a necessidade de utilização de dados de bases diferentes para suas concepções. Contudo, a junção das bases foi desenvolvida com a atenção necessária para não prejudicar os resultados. Vejamos na seção seguinte os resultados obtidos e, então, a validade das proxies utilizadas.

\section{Resultados}

Os resultados apontam um painel equilibrado, ou fortemente balanceado. Isso decorre do fato de todas as unidades consideradas serem estudadas sob a mesma série temporal em todas as variáveis, o período de 2005 a 2014. Resultado já esperado tendo em vista o apontamento feito na seção anterior. Para fins de melhor explanação dos resultados dividiu-se as análises em duas subseções. A primeira trata da estatística descritiva e a segunda realiza a análise do modelo estimado.

\subsection{Estatística descritiva}

Inicialmente, e de forma geral, tem-se um exercício básico de estatística descritiva no qual se apresenta a média, o desvio padrão e os valores de mínimo e de máximo para todas as variáveis e no período completo da análise. Tais resultados apresentam-se dispostos na tabela 1, abaixo: 
Tabela 1: Estatística descritiva da amostra completa: estados brasileiros, de 2005 a 2014.

\begin{tabular}{cc|c|c|c|c}
\hline VARIÁVEL & & MÉDIA & DESV.PAD. & MÍN. & MÁX. \\
\hline \multirow{2}{*}{ PIB } & Overall & 106.372 & $185.411,6$ & 3.179 & 1.188 .091 \\
& Between & & $185.570,4$ & $4.736,43$ & $944.307,1$ \\
& Within & & $33.064,4$ & $-110.951,1$ & 350.156 \\
CAP_FIS & Overall & $11.235,02$ & $17.994,50$ & 192,06 & $97.440,32$ \\
& Between & & $18.222,95$ & 276,66 & $89.575,59$ \\
& Within & & $1.684,18$ & $1.000,01$ & $19.099,75$ \\
CAP_HUM & Overall & 27,39 & 5,55 & 14,82 & 40,37 \\
& Between & & 4,87 & 18,89 & 35,86 \\
& Within & & 2,80 & 20,63 & 34,99 \\
TRABALHO & Overall & 4.349 .288 & 5.195 .313 & $249.571,8$ & $2,67 \mathrm{e}+07$ \\
& Between & & 5.282 .810 & $270.040,7$ & $2,59 \mathrm{e}+07$ \\
& Within & & $130.081,2$ & 3.802 .699 & 5.138 .019 \\
\hline
\end{tabular}

Fonte: Elaboração dos autores fundamentada nos resultados gerados pelo STATA.

Legenda: Overall: Toda a amostra; Between: Entre as unidades; Within: Dentro da unidade.

Apesar de essa abordagem proporcionar uma análise simplificada, baseada na Tabela 1 é possível extrair informações importantes sobre o comportamento das variáveis. Os valores médios apresentam uma estatística resumida de forma tal que condensam informações na medida em que especificidades possam ser desconsideradas. Entretanto, deve-se atentar para a unidade de medida das variáveis, tais como descritas anteriormente, na seção de Metodologia.

Juntando as análises mencionadas acima nota-se uma diferença significativa entre os valores de mínimo e de máximo vis-à-vis o termo médio. Da análise principal deste estudo, a lembrar, capital humano e crescimento econômico, note a diferença entre o mínimo do PIB - aproximadamente 3,2 bilhões - e o PIB médio - 106 bilhões -. E ainda, deste para o PIB máximo, quase 1,2 trilhões. Já a variável de capital humano apresenta média de 27,3, tendo 14,8 de mínimo e 40,3 de máximo. Diferenças consideráveis nessa variável explicativa que, como veremos adiante, impactam no desempenho do PIB da forma apresentada no início do parágrafo. Comparações outras ficam a cargo do leitor.

Uma vez que o desvio padrão é uma medida de dispersão absoluta e, como tal, apresenta limitações analíticas vis-à-vis medidas relativas. Aos interessados, dispôsse uma tabela em anexo (Tabela 4) considerando o coeficiente de variação de Pearson das variáveis PIB e Capital Humano para todos os estados brasileiros. Isso possibilita uma melhor comparação entre todas as unidades por tratarem em termos das taxas. 
Embora o trabalho contemple outras variáveis, dado o enfoque em analisar o capital humano e o crescimento econômico para os estados brasileiros viu-se, pois, como interessante dispor dados descritivos sobre as variáveis PIB e CAP_HUM desagregados para as unidades estudadas. Assim, o gráfico a seguir apresenta tais informações para todos os estados brasileiros.

Gráfico 1: Estatística descritiva para as variáveis PIB e CAP_HUM* para os estados brasileiros.

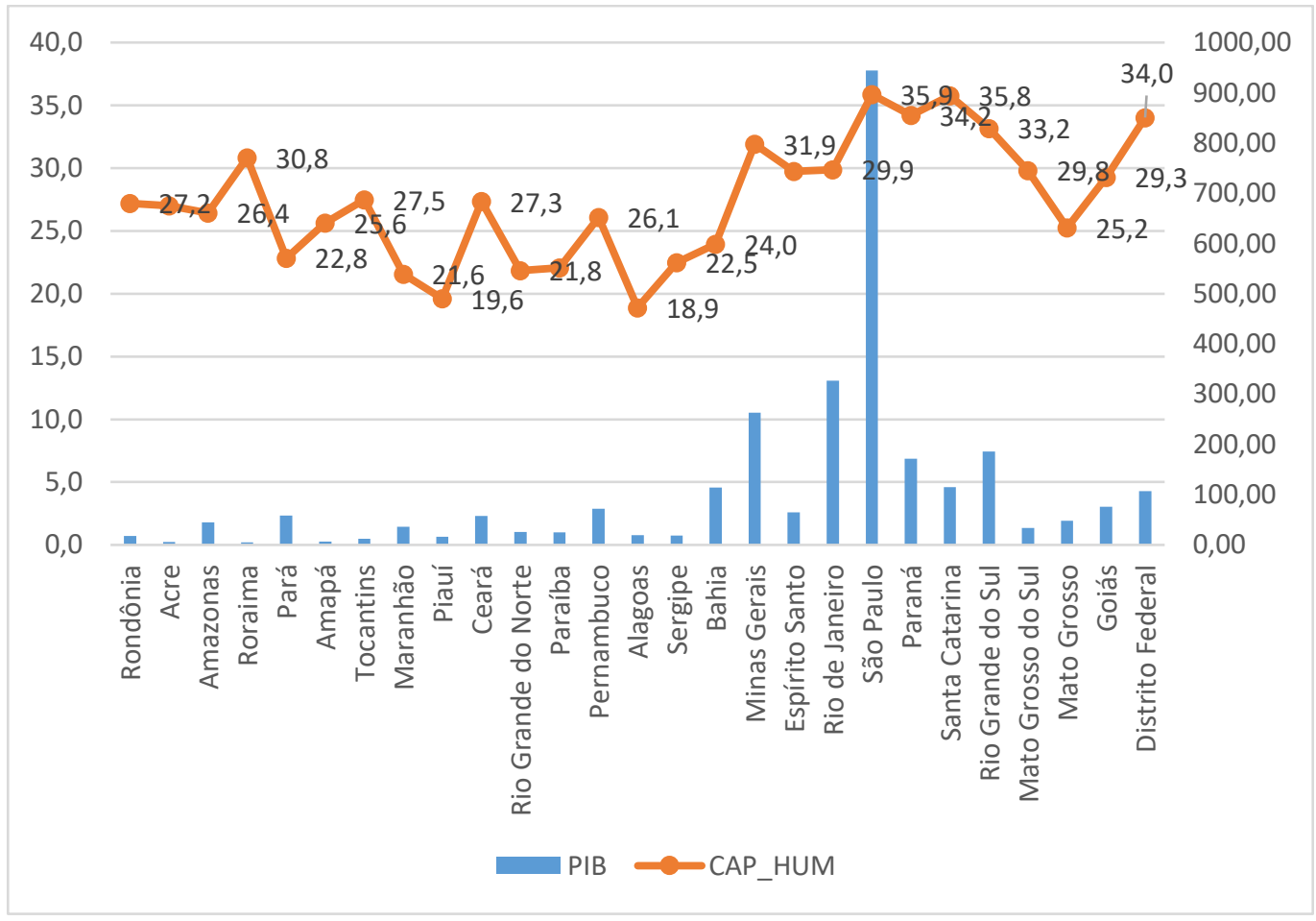

Fonte: elaboração própria. Dados das séries especificadas na seção anterior. Nota: PIB em bilhões.

Nota: Valores referentes à média do período estudado (2005 a 2014).

A análise do gráfico acima deve ser realizada com cautela. Os estados pertencentes aos grupos de menor PIB, não necessariamente os que possuem menor PIB, também possuem menor estoque de capital humano. Esse exercício pode ser realizado por meio da divisão de regiões geográficas. Isto se deve, obviamente, aos outros fatores da função de produção considerada no modelo. 0 gráfico dispõe apenas das sequências de estados por região sem, no entanto, explicitar uma clara divisão delas. 
Analisando os estados da região Nordeste, por exemplo, percebe-se que a média de capital humano flutua em torno de 21, independentemente do nível do PIB, e todos eles abaixo daqueles estados das regiões Sul e Sudeste. Nesse comparativo destaca-se Ceará e Pernambuco com 27 e 26, respectivamente, por mais se aproximarem da média do Sul e Sudeste. Entretanto, se compararmos os máximos alcançados pelas séries de cada estado temos São Paulo com 40 e Pernambuco com 33,5. Tais comparações são arbitrárias e objetivam apenas exemplificação. Novamente, exercícios outros ficam a cargo do leitor.

Porém, aqueles com maior PIB geralmente apresentam maiores médias de capital humano. Nesse caso a atenção deve voltar-se aos estados da região Sul e Sudeste. Estes apresentam níveis de PIB elevados, na casa das centenas de bilhões com destaque para São Paulo que em seu máximo atinge a casa dos trilhões (informação não exposta no gráfico ${ }^{15}$ ) e os níveis de capital humano flutuam ao redor de 32, onde se destacam São Paulo e Santa Catarina com máximos de 40 e 39,7, respectivamente.

\subsection{Modelo estimado}

Modelos de dados em painel são uma junção de séries temporais com cortes transversais e podem apresentar características de ambas as formas. Séries temporais geralmente apresentam um coeficiente de determinação alto e apontamse como resultado de correlação serial. Já dados de cortes transversais geralmente apresentam heterocedasticidade. Segundo Gujarati $(2006$, p. 316) "a assimetria na distribuição de um ou mais regressores considerados no modelo é uma fonte de heterocedasticidade, pois variáveis como renda e escolaridade apresentam desigualdades na maioria das sociedades". Vê-se, pois, certa semelhança com o presente estudo, tanto das variáveis como das unidades analisadas.

Pelos testes de Wooldridge e de Wald detectaram-se, respectivamente, a presença de correlação serial e de heterocedasticidade. 0 teste de Hausman, para escolha entre os modelos de efeitos fixos e de efeitos aleatórios, apontou para efeitos fixos. 0 teste de Chow para escolha entre modelos de efeitos fixos e Pooled apontou também para efeitos fixos. A tabela a seguir mostra uma comparação entre os modelos considerados a priori.

Muito embora o teste de Hausman tenha apontado para efeitos fixos deve-se ter em mente que os testes são apenas indicativos, não sendo, portanto, critério exclusivo para escolha do método. Adicionalmente, é compreensível e notório que se percebam especificidades (heterogeneidade não-observada) entre as regiões brasileiras e de seus estados em particular. Assim, visando abordar a heterogeneidade das unidades e corrigir a heterocedasticidade e a autocorrelação, optou-se por estimar pelo método dos mínimos quadrados generalizados (GLS) com efeitos aleatórios, (conforme a equação (6)).

15 Aos interessados, a Tabela (3) em anexo contém informações adicionais para todos os estados brasileiros. 
Considerar somente os testes mencionados acima e/ou os coeficientes e suas respectivas significâncias estatísticas não necessariamente é suficiente para a escolha do modelo mais apropriado. Do contrário, pela Tabela 2 abaixo que comporta os resultados de todos os modelos considerados, seríamos praticamente indiferentes entre eles.

As análises basear-se-ão no modelo com efeitos aleatórios estimado com GLS, tal como argumentado acima. Percebe-se, com base na Tabela 2, que o modelo utilizado se mostrou bem ajustado e que todas as variáveis utilizadas como explicativas apresentaram significância estatística verificada pelo $p$-valor. Em particular, a variável de capital humano, foco deste estudo, com intervalo de confiança de $99 \%$.

Tabela 2: Estimação dos diferentes modelos

\begin{tabular}{cccc}
\hline VARIÁVEL & EA (GLS) & EF & POOLED (GLS) \\
\hline Log CAP_FIS & $0,58338297^{* * *}$ & $0,61871404^{* * *}$ & $0,48276844^{* * *}$ \\
Log CAP_HUM & $0,8656655^{* * *}$ & $0,7822207^{* * *}$ & $1,3257256^{* * *}$ \\
Log TRABALHO & $0,3268012^{*}$ & $0,56689705^{*}$ & $0,41268052^{* * *}$ \\
_cons & $-1,84096$ & $-5,40733$ & $-3,79649^{* * *}$ \\
\hline
\end{tabular}

Fonte: Elaboração dos autores com base nos resultados gerados pelo Stata

Legenda: EA: Efeitos Aleatórios; EF: Efeitos Fixos; GLS: Mínimos Quadrados Generalizados. Nota: ${ }^{*} \mathrm{p}<0,1 ;{ }^{* *} \mathrm{p}<0,05 ;{ }^{* * *} \mathrm{p}<0,01$.

Verifica-se, ainda, os sinais positivos dos coeficientes. Fato já esperado, tendo em vista que são fatores da função de produção especificada na seção de metodologia. Vê-se, pois, que todas as variáveis consideradas, a lembrar, capital físico, capital humano e trabalho, relacionam-se positivamente com a variável dependente, PIB.

Nota-se, pela soma dos coeficientes estimados, que a equação estimada apresenta retornos crescentes de escala. Destaca-se o coeficiente do capital humano, principal atenção deste estudo, com valor aproximado de 0,87. Esse valor pode se dever às externalidades geradas pelo capital humano. Além das externalidades mencionadas na seção de referencial teórico, destaca-se Shleifer (1990) argumentando que o capital humano impacta positivamente na produtividade e nos salários implicando em um aumento consequente da demanda. Com isso a associação com o PIB torna-se trivial. 
0 resultado apresentado acima corrobora tanto a teoria dos modelos de crescimento, sobretudo aqueles sobre capital humano, foco deste trabalho, quanto a quase totalidade dos trabalhos dessa área apresentados ao longo deste trabalho.

\section{Considerações finais}

0 presente trabalho analisou o crescimento do PIB dos estados brasileiros no período anual de 2005 a 2014 utilizando, para tanto, o modelo de Mankiw, Romer e Weil (1992). Dada a natureza do trabalho utilizou-se modelos de dados em painel. 0 trato da heterogeneidade das unidades e a necessidade de correção da heterocedasticidade e a autocorrelação detectadas influenciou a escolha de estimar pelo método dos mínimos quadrados generalizados (GLS) com efeitos aleatórios. Atenta-se ao fato de que a correção desses problemas ajuda a contornar, inclusive, um provável problema de endogeneidade.

Os sinais e as magnitudes dos coeficientes dentro da normalidade, ou não díspares da literatura afim, considerando a forma como tal modelo foi estimado, mostraram que o modelo adotado com os fatores capital físico, capital humano e trabalho foi adequado para o objetivo proposto.

Analisando os fatores considerados, obteve-se que as proxies utilizadas - Consumo de energia não residencial, para capital físico; Anos de estudo da PEA multiplicado pelo Ideb referente ao ensino médio, para capital humano; e População ocupada, para trabalho - mostraram-se boas representações das variáveis, tendo em vista os resultados obtidos.

Considerando uma análise particularizada do fator capital humano, objetivo do presente trabalho, e o foco na qualidade da educação básica, tendo em vista que a proxy utilizada contém o Ideb, um índice que representa a qualidade na educação básica no Brasil, pode-se afirmar, com base nos resultados e na literatura citada, que a qualidade na educação é um importante fator para aumento do PIB, no período e unidades avaliados.

Os resultados obtidos aqui, mais especificamente, acerca da importância da qualidade da educação como capital humano se somam a vários outros, tanto nacionais quanto internacionais, bem como à teoria do capital humano pautada no meio acadêmico há décadas, e contribui para a concretização da evidência da relação positiva e significativa entre capital humano e crescimento econômico. Além disso, contribui por meio da especificidade gerada pelo recorte da pesquisa, pelo período e pelas proxies utilizadas.

Dada a amplitude da área de estudo, que envolve educação e crescimento, o presente estudo, além dos resultados obtidos, gerou curiosidades em relação a outros enfoques. Um enfoque imediato é a realização de comparações entre a proxy de capital humano utilizada e outras que envolvam qualidade na educação. Isso pode ser feito a fim de munir os interessados dessa área sobre quais proxies utilizar visando maior acurácia em suas respectivas análises, considerando, claro, as especificidades de cada pesquisa.

Em suma, atingiu-se o objetivo esperado de demonstrar a importância do capital humano, considerando a qualidade da educação básica, no crescimento econômico dos 
estados brasileiros. Com isso, espera-se chamar atenção para a mudança do enfoque de quantitativo, esboçado pelo governo brasileiro ao longo do tempo, para uma preocupação maior com a qualidade da educação básica.

\section{Referências}

AGHION, Philippe; HOWITT, Peter. Endogenous Growth Theory. 3 ed. The MIT Press. Massachusetts Institute of Technology: Cambridge, Massachusetts, 1999.

BARBOSA FILHO, Fernando de Holanda; PESSÔA, Samuel. Educação, crescimento e distribuição de renda: A experiência brasileira em perspectiva histórica. In:

Educação básica no Brasil: construindo um país do futuro. VELOSO, Fernando. et. al. Elsevier: Rio de Janeiro, 2009.

BARBOSA FILHO, Fernando de Holanda; PESSÔA, Samuel. Educação e desenvolvimento no Brasil. In: Desenvolvimento Econômico: Uma perspectiva Brasileira. FERREIRA, Pedro et. al. Elsevier: Rio de Janeiro, 2013.

BARRETTO, E. S. de S.; MITRULIS, E. Trajetória e desafios dos ciclos escolares no País. Revista Estudos Avançados USP, p. 1-39: São Paulo, 2001. DOI: https://doi.org/10.1590/S0103-40142001000200003

BARRO, Robert. Economic growth in a cross section of countries. The quarterly journal of economics. Vol. 106, №. 2. The MIT Press: Massachusets, 1991. DOI: https://doi.org/10.2307/2937943

BARRO, Robert J. Education and economic growth. Annals of economics and finance. Harvard Universit. 2013.

BARROS, Alexandre Hands. Desigualdades regionais e desenvolvimento econômico. In: Desenvolvimento Econômico: Uma perspectiva Brasileira. FERREIRA, Pedro et. al. Elsevier: Rio de Janeiro, 2013.

BENHABIB, Jess; SPIEGEL, Mark M. The role of human capital in economic development evidence from aggregate cross-country data. Department of Economics, New York University. Journal of Monetary, n.34, p. 143-173, Elsevier: New York, 1994. DOI: https://doi.org/10.1016/0304-3932(94)90047-7

BRASIL. ANUÁRIO ESTATÍSTICO DE ENERGIA ELÉTRICA 2011. Empresa de Pesquisa Energética, Ministério de Minas e Energia. Rio de Janeiro, 2011. 
BRASIL. ANUÁRIO ESTATÍSTICO DE ENERGIA ELÉTRICA 2013. Empresa de Pesquisa Energética, Ministério de Minas e Energia. Rio de Janeiro, 2013.

BRASIL. ANUÁRIO ESTATÍSTICO DE ENERGIA ELÉTRICA 2015 (ano base 2014). Empresa de Pesquisa Energética, Ministério de Minas e Energia. Rio de Janeiro, 2015.

CONNOLLY, M. P. Human capital and growth in the Post-Bellum South: a separate but unequal story. The Journal of Economic History, n. 64, p. 1-39. 2004. DOI: https://doi.org/10.1017/S0022050704002736

FIRME, Vinícius de A. Couto; SIMÃo FILHO, José. Análise do crescimento econômico dos municípios de Minas Gerais através do modelo de Solow ampliado, com inserção de capital Humano e condições de saúde, 1991-2000: um estudo de caso para econometria espacial. Anais do XLII Encontro Nacional de Economia. Associação Nacional dos Centros de Pós-Graduação em Economia - ANPEC, 2014. DOI: https://doi.org/10.1590/1413-8050/ea640

FIRME, Vinícius de A. Couto; FREGUGLIA, Ricardo da Silva. Análise do crescimento dos municípios brasileiros utilizando dados em painel e controles espaciais sobre o modelo de Mankiw, Romer e Weil (1992) para o período de 1980 a 2010. Anais do XLI Encontro Nacional de Economia. ANPEC, 2013.

GROSSMAN, Michael. Education and nonmarket outcomes. In: HANUSHEK, E.A.; WELCH, F. Handbook of the economics of education, North-Holland, 2006. DOI: https://doi.org/10.1016/S1574-0692(06)01010-5

GUJARATI, Damodar. Econometria Básica. 4 ed. Elsevier / Campus: Rio de janeiro, 2006.

HANUSHEK, Eric; KIMKO, Dennis. Schooling, Labor Force Quality, and the Growth of Nations. The American Economic Review. Vol. 90, № 5, p. 1184-1208. 2000. DOI: https://doi.org/10.1257/aer.90.5.1184

HILL, R. Carter; GRIFFITHS, William E.; JUDGE, George G. Econometria. Trad. Alfredo A. de Farias. São Paulo: Saraiva, 1999.

HSIAO, Cheng. Analysis of panel data. Cambridge: Cambridge University Press, 1986.

INSTITUTO BRASILEIRO DE GEOGRAFIA E ESTATÍSTICA - IBGE. Contas regionais do Brasil: 2005 - 2009. Contas Nacionais, n. 35. Coordenador: Roberto Luis Olinto Ramos; Rio de Janeiro, 2011. 
INSTITUTO BRASILEIRO DE GEOGRAFIA E ESTATÍSTICA - IBGE. Pesquisa Nacional por Amostra de Domicílio - PNAD: 2005 - 2013. 2014a. Disponível em:

www.ibge.gov.br; Acesso em: 10 de setembro de 2016.

INSTITUTO BRASILEIRO DE GEOGRAFIA E ESTATÍSTICA - IBGE. Contas Regionais do Brasil: 2010 - 2012. 2014b. Disponível em: www.ibge.gov.br; Acesso em: 09 de setembro de 2016

INSTITUTO BRASILEIRO DE GEOGRAFIA E ESTATÍSTICA - IBGE. Contas Regionais do Brasil: 2010 - 2014. 2016. Disponível em: https://www.ibge.gov.br/ estatisticasnovoportal/economicas/contas-nacionais/9054-contas-regionais-dobrasil.html?edicao=9055\&t=publicacoes. Acesso em: 05 de novembro de 2016 .

KENNEDY, Peter. A guide to econometrics. Massachusetts: MIT Press Google Scholar, 2008.

LEME, Maria Carolina; PAREDES, Ricardo; SOUZA, André Portela. A municipalização do ensino fundamental e seu impacto sobre a proficiência no Brasil. In: Educação básica no Brasil: construindo um país do futuro. VELOSO, Fernando. et. al. Elsevier: Rio de Janeiro, 2009.

LUCAS, Robert E. On the machanics of economic growth. University of Chicago. Chicago, 1988.

MANKIW, N. Gregory; ROMER, David; WEIL, David N. A contribuition to the empirics of economic growth. The Quarterly Journal of Economics, v. 107, n. 2, p. 407-437. The MIT Press: Massachucets, 1992. DOI: https://doi.org/10.2307/2118477

MARQUETTI, Adalmir A.; BERNI, Duílio de Ávila; HICKMANN, Gustavo. Evidências empíricas sobre a relação entre educação e crescimento no Rio Grande do Sul. Indicadores Econômicos FEE. v. 30, n. 2, p. 105-122: Porto Alegre, 2002.

NAKABASHI, Luciano; FIGUEIREDO, Lízia de. Capital Humano: uma nova proxy para incluir aspectos qualitativos. Revista de Economia. Ano 32, v. 34, n. 1, p. 7-24. Editora UFPR: Belém, 2008. https://doi.org/10.5380/re.v34i1.5981

NAKABASHI, Luciano; FIGUEIREDO, Lízia de. Capital humano e crescimento: impactos diretos e indiretos. Anais do XXXIII Encontro Nacional de Economia. Associação Nacional dos Centros de Pós-Graduação em Economia - ANPEC, 2005. 
NELSON, Richard R.; PHELPS, Edmund S. Investment in humans, technological diffusion and economic growth. The American Economic Review, v. 56, n. 1/2. p. 66-75. American Economic Association, 1966.

PEREIRA, André da Silva. Uma análise do capital humano e crescimento econômico brasileiro no período de 1970 a 2001. Programa de Pós-Graduação em Economia. Faculdade de Ciência Econômicas - Universidade Federal do Rio Grande do Sul: Porto Alegre, 2004.

PESSÔA, Samuel de Abreu. Perspectivas de crescimento no longo prazo para o Brasil: questões em aberto. Ensaios Econômicos. Escola de Pós-graduação em Economia da Fundação Getúlio Vargas: Rio de Janeiro, 2006.

ROMER, Paul M. Increasing returns and long-run growth. Journal of Political Economy. v. 94, n. 5, p. 1002-1037. The University of Chicago Press: Chicago, 1986. DOI: https://doi.org/10.1086/261420

SALA-I-MARTIN, Xavier. Apuntes de crecimiento econômico. Antoni Bosch, editor. 2000.

SCHULTZ, Theodore W. Investment in human capital. The American Economic Review. v. 51, n. 1, p. 1-17. American Economic Association, 1961.

SHLEIFER, Andrei. Externalidades como motor do crescimento. Revista Brasileira de Economia. V. 44, n. 3. 1990. Disponível em: http://bibliotecadigital.fgv.br/ojs/ index.php/rbe/article/viewFile/457/6646 Acesso em: 19 de agosto de 2016.

SOARES, C. M. M. Nível de renda e a qualidade da educação nos municípios brasileiros. Monografias PET, UFMG: Minas Gerais, 2009.

SOLOW, Robert M. A contribuition to the theory of economic growth. The Quarterly Journal of Economics. v. 70, n. 1. The MIT Press: Massachusets, 1956. DOI: https://doi.org/10.2307/1884513

TAVARES, Jean Max; ATALIBA, Flávio; CASTELAR, Ivan. Mensuração da produtividade total dos fatores para os estados brasileiros, sua contribuição ao crescimento do produto e influência da educação. Revista Econômica do Nordeste, v. 32, n. Especial, p. 633-653: Fortaleza, 2001. 
TEMPLE, Jonathan. A positive effect of human capital on growth. Institute of Economics and Statistics, Oxford University. Economics Letters, n. 65, p. 131-134: Elsevier, 1999. DOI: https://doi.org/10.1016/S0165-1765(99)00120-2

TEMPLE, Jonathan. Growth effects of education and social capital in the OCDE countries. OCDE Economic Studies, n. 33. 2001. 


\section{ANEXO A}

Tabela 3: Estatística descritiva para as variáveis PIB e CAP_HUM para os estados brasileiros

\begin{tabular}{|c|c|c|c|c|c|}
\hline ESTADO & VARIÁVEL & MÉDIA & DESV. PAD. & MÍNIMO & MÁXIMO \\
\hline \multirow[t]{2}{*}{ Rondônia } & PIB & $17.466,30$ & $3.499,97$ & $12.707,75$ & $21.710,88$ \\
\hline & CAP_HUM & 27,21 & 3,29 & 22,12 & 30,84 \\
\hline \multirow[t]{2}{*}{ Acre } & PIB & $6.231,72$ & $1.160,84$ & $4.483,00$ & $8.040,49$ \\
\hline & CAP_HUM & 26,99 & 2,86 & 21,63 & 29,23 \\
\hline \multirow[t]{2}{*}{ Amazonas } & PIB & $44.753,92$ & $7.832,78$ & $33.352,00$ & $58.506,65$ \\
\hline & CAP_HUM & 26,44 & 3,95 & 19,69 & 31,40 \\
\hline \multirow[t]{2}{*}{ Roraima } & PIB & $4.736,43$ & $1.038,19$ & 3.179 & $6.428,64$ \\
\hline & CAP_HUM & 30,83 & 2,96 & 26,55 & 35,25 \\
\hline \multirow[t]{2}{*}{ Pará } & PIB & $58.785,99$ & $16.061,06$ & 39.121 & $87.400,57$ \\
\hline & CAP_HUM & 22,84 & 2,45 & 19,02 & 25,58 \\
\hline \multirow[t]{2}{*}{ Amapá } & PIB & $6.537,44$ & $1.463,78$ & 4.361 & $9.122,13$ \\
\hline & CAP_HUM & 25,63 & 2,20 & 23,16 & 28,37 \\
\hline \multirow[t]{2}{*}{ Tocantins } & PIB & $12.583,63$ & $2.601,49$ & 9.061 & $16.775,42$ \\
\hline & CAP_HUM & 27,48 & 3,16 & 22,22 & 31,27 \\
\hline \multirow[t]{2}{*}{ Maranhão } & PIB & $35.849,02$ & $7.399,49$ & 25.335 & $47.763,31$ \\
\hline & CAP_HUM & 21,56 & 2,81 & 15,77 & 23,45 \\
\hline \multirow[t]{2}{*}{ Piauí } & PIB & $16.423,73$ & $3.625,60$ & 11.129 & $22.216,10$ \\
\hline & CAP_HUM & 19,62 & 3,25 & 14,82 & 24,21 \\
\hline \multirow[t]{2}{*}{ Ceará } & PIB & $58.034,05$ & $11.962,34$ & $40.935,00$ & $76.877,68$ \\
\hline & CAP_HUM & 27,34 & 2,48 & 23,19 & 29,77 \\
\hline \multirow[t]{2}{*}{ Rio Grande do Norte } & PIB & $25.614,42$ & $6.055,03$ & 17.870 & $36.721,45$ \\
\hline & CAP_HUM & 21,84 & 1,84 & 19,46 & 24,15 \\
\hline \multirow[t]{2}{*}{ Paraíba } & PIB & $24.609,99$ & $4.998,70$ & 16.869 & $32.839,54$ \\
\hline & CAP_HUM & 22,07 & 2,61 & 17,72 & 25,28 \\
\hline \multirow[t]{2}{*}{ Pernambuco } & PIB & $71.862,05$ & $16.909,25$ & 49.922 & $100.101,70$ \\
\hline & CAP_HUM & 26,08 & 4,41 & 21,41 & 33,47 \\
\hline \multirow[t]{2}{*}{ Alagoas } & PIB & $19.339,30$ & $3.972,40$ & 14.139 & $26.274,13$ \\
\hline & CAP_HUM & 18,89 & 1,19 & 17,22 & 20,37 \\
\hline
\end{tabular}




\begin{tabular}{|c|c|c|c|c|c|}
\hline ESTADO & VARIÁVEL & MÉDIA & DESV. PAD. & MÍNIMO & MÁXIMO \\
\hline \multirow[t]{2}{*}{ Sergipe } & PIB & $18.383,27$ & $3.655,77$ & 13.427 & $24.845,89$ \\
\hline & CAP_HUM & 22,49 & 1,57 & 20,24 & 24,01 \\
\hline \multirow[t]{2}{*}{ Bahia } & PIB & $114.457,20$ & $16.623,78$ & $90.919,00$ & $141.319,60$ \\
\hline & CAP_HUM & 23,96 & 2,11 & 20,49 & 26,16 \\
\hline \multirow[t]{2}{*}{ Minas Gerais } & PIB & $262.974,60$ & $48.782,88$ & 192.639 & $342.120,70$ \\
\hline & CAP_HUM & 31,90 & 2,22 & 28,33 & 34,42 \\
\hline \multirow[t]{2}{*}{ Espírito Santo } & PIB & $64.504,31$ & $12.043,95$ & 47.223 & $82.076,67$ \\
\hline & CAP_HUM & 29,74 & 2,16 & 26,65 & 32,51 \\
\hline \multirow[t]{2}{*}{ Rio de Janeiro } & PIB & $326.596,90$ & $63.284,79$ & 247.018 & $439.976,10$ \\
\hline & CAP_HUM & 29,87 & 4,30 & 25,79 & 37,19 \\
\hline \multirow[t]{2}{*}{ São Paulo } & PIB & $944.307,10$ & $147.328,70$ & 726.984 & 1.188 .091 \\
\hline & CAP_HUM & 35,86 & 3,50 & 31,02 & 40,37 \\
\hline \multirow[t]{2}{*}{ Paraná } & PIB & $171.285,50$ & $34.910,06$ & 126.677 & $235.020,80$ \\
\hline & CAP_HUM & 34,20 & 2,62 & 29,48 & 37,04 \\
\hline \multirow[t]{2}{*}{ Santa Catarina } & PIB & $115.413,50$ & $21.436,48$ & 85.316 & $150.278,10$ \\
\hline & CAP_HUM & 35,76 & 2,46 & 31,84 & 39,71 \\
\hline \multirow[t]{2}{*}{ Rio Grande do Sul } & PIB & $185.816,10$ & $27.875,24$ & 144.218 & $229.635,10$ \\
\hline & CAP_HUM & 33,16 & 1,98 & 30,89 & 36,37 \\
\hline \multirow[t]{2}{*}{ Mato Grosso do Sul } & PIB & $33.637,92$ & $9.317,21$ & 21.651 & $49.955,32$ \\
\hline & CAP_HUM & 29,82 & 3,60 & 23,06 & 32,81 \\
\hline \multirow[t]{2}{*}{ Mato Grosso } & PIB & $48.505,04$ & $9.468,05$ & $34.184,01$ & $62.257,42$ \\
\hline & CAP_HUM & 25,24 & 2,54 & 20,96 & 28,65 \\
\hline \multirow[t]{2}{*}{ Goiás } & PIB & $76.031,40$ & $18.961,18$ & 50.534 & $108.169,10$ \\
\hline & CAP_HUM & 29,27 & 5,24 & 23,82 & 36,87 \\
\hline \multirow[t]{2}{*}{ Distrito Federal } & PIB & 107.302 & $15.569,61$ & 80.527 & 122.521 \\
\hline & CAP_HUM & 34,03 & 2,05 & 30,69 & 37,07 \\
\hline \multirow[t]{2}{*}{ Brasil } & PIB & 106.372 & $185.411,6$ & 3.179 & 1.188 .091 \\
\hline & CAP_HUM & 27,39 & 5,55 & 14,82 & 40,37 \\
\hline
\end{tabular}

Fonte: Elaboração dos autores a partir dos resultados gerados pelo STATA. 


\section{ANEXO B}

Tabela 4: Coeficiente de variação de Pearson do PIB e CAP_HUM:

Estados brasileiros de 2005 a 2014

\begin{tabular}{|c|c|c|c|}
\hline ESTADO & PPIB*|PCAP_HUM** & ESTADO & PPIB*|PCAP_HUM** \\
\hline \multirow[t]{2}{*}{ Rondônia } & 20,04 & Maranhão & 20,64 \\
\hline & 12,08 & & 13,02 \\
\hline \multirow[t]{2}{*}{ Acre } & 18,63 & Piauí & 22,08 \\
\hline & 10,60 & & 16,57 \\
\hline \multirow[t]{2}{*}{ Amazonas } & 17,50 & Ceará & 20,61 \\
\hline & 14,93 & & 9,07 \\
\hline \multirow[t]{2}{*}{ Roraima } & 21,92 & Rio Grande do Norte & 23,64 \\
\hline & 9,60 & & 8,41 \\
\hline \multirow[t]{2}{*}{ Pará } & 27,32 & Paraíba & 20,31 \\
\hline & 10,71 & & 11,84 \\
\hline \multirow[t]{2}{*}{ Amapá } & 22,39 & Pernambuco & 23,53 \\
\hline & 8,58 & & 16,90 \\
\hline \multirow[t]{2}{*}{ Tocantins } & 20,67 & Alagoas & 20,54 \\
\hline & 11,50 & & 6,28 \\
\hline \multirow[t]{2}{*}{ Paraná } & 20,38 & Sergipe & 19,89 \\
\hline & 7,67 & & 6,97 \\
\hline \multirow[t]{2}{*}{ Santa Catarina } & 18,57 & Bahia & 14,52 \\
\hline & 6,87 & & 8,79 \\
\hline \multirow[t]{2}{*}{ Rio Grande do Sul } & 15,00 & Minas Gerais & 18,55 \\
\hline & 5,96 & & 6,95 \\
\hline \multirow[t]{2}{*}{ Mato Grosso do Sul } & 27,70 & Espírito Santo & 18,67 \\
\hline & 12,07 & & 7,27 \\
\hline \multirow[t]{2}{*}{ Mato Grosso } & 19,52 & Rio de Janeiro & 19,38 \\
\hline & 10,08 & & 14,40 \\
\hline \multirow[t]{2}{*}{ Goiás } & 24,94 & São Paulo & 15,60 \\
\hline & 17,89 & & 9,75 \\
\hline \multirow[t]{2}{*}{ Distrito Federal } & 14,51 & Brasil & 174,30 \\
\hline & 6,02 & & 20,27 \\
\hline
\end{tabular}

Fonte: Elaboração dos autores a partir dos resultados gerados pelo STATA**.

*PPIB: Coeficiente de variação de Pearson do PIB.

**PCAP_HUM: Coeficiente de variação de Pearson do CAP_HUM.

***O STATA gerou apenas os valores referentes a média e desvio padrão, o coeficiente de variação de Pearson foi calculado pelos autores. 